\title{
Infantile Nephropathic Cystinosis in Sulaimani Pediatric Teaching Hospital: A Retrospective Cohort Study
}

\author{
Hunar Jamal Hussein \\ MBChB, KBMS Trainee \\ Pediatrics \\ Kurdistan Board for Medical Specialties \\ Erbil, Iraq \\ hunarjamal85@yahoo.com
}

\author{
Khalid Hama Salih \\ MBChB, DCH, FIBMS Ped., Lecturer \\ Pediatrics \\ School of Medicine \\ University of Sulaimani \\ Sulaimani, Iraq \\ Khalidh976@yahoo.com
}

\author{
Adnan Mohammed Hasan \\ Professor \\ Pediatrics \\ School of Medicine \\ University of Sulaimani \\ Sulaimani, Iraq \\ dr.adnanhasan@yahoo.com
}

\begin{abstract}
Cystinosis is a rare metabolic autosomal recessive disorder which characterized by intralysosomal accumulation of cystine. There are three forms; infantile nephropathic is the commonest forms. to evaluate clinical presentations and outcome of infantile cystinosis. A retrospective cohort study conducted in Sulaimani Pediatric Teaching Hospital on 25 patients with infantile cystinosis during May 1, 2014, to June 1, 2017. This study has depended on clinical symptoms and signs, and corneal crystallization for the diagnosis of cystinosis. Gender of the patients was 13 (52\%) females and 12 (48\%) males. The ages were ranged between (1-12 years) with a mean age of (6.25 years). Eight (32\%) patients were from Sulaimani city, but the other 17 (68\%) patients were from outside of Sulaimani. Moreover, a 17 (68\%) of them were Arabic and the other eight (32\%) were Kurdish ethnic groups. The study showed a 20 (80\%) positive consanguinity with 19 (76\%) positive family history of infantile cystinosis. Additionally, the age of first presentations was between (0.252 years) with a mean of ( 0.8 years). Clinical features included a $100 \%$ for polyuria, polydipsia, and failure to thrive. Furthermore, $10(40 \%)$ presented with constipation, 23 (92\%) photophobia and 5 (20\%) blond hair. Complications included 24 (96\%) rickets, 14 (56\%) renal insufficiency, 5 (20\%) hypothyroidism, 4 (16\%) genu valgum, 3 (12\%) growth hormone deficiency, and 3 (12\%) developed end-stage renal disease. Subsequently, two patients died (8\%) due to endstage renal disease. Finally, there was a statistically significant relationship between both renal insufficiency $(P$ value $=0.042)$ and hypothyroidism $(P$-value $<0.001)$ with Kurdish ethnicity. Conclusion: Incidence of cystinosis was high among consanguineous parents and those patients who had a positive family history of cystinosis. Furthermore, the delay in diagnosis was due to atypical presentations and unavailability of specific investigations.
\end{abstract}

Keywords: infantile cystinosis, clinical presentations, consanguinity, family history.

\section{INTRODUCTION:}

Cystinosis is a rare autosomal recessive metabolic disease [1] that characterized by cystine accumulation inside lysosome because of a defect in transport system of cystine [2].

There are three types of cystinosis which are as follows [3]:

1. Infantile nephropathic cystinosis: it is the severe and most common type which make infant to suffer from symptoms and signs of Fanconi syndrome during the first year of their life.

2. Juvenile nephropathic cystinosis: it presents with proteinuria and a mild form of Fanconi syndrome.

3. Ocular cystinosis: this type is rare before adulthood [1] and it is characterized by cystine deposition in the cornea and conjunctiva.

The cause of all types of cystinosis is by CTNS (cystinosin, lysosomal cystine transporter) gene mutation on the short-arm of chromosome number 17. Furthermore, it Has been mentioned in the literatures that more than 80 mutations are present [4]. The prevalence is 1 in 100,000 - 200,000 [5] and the highest prevalence was found among the Pakistani group who lives in the west of the United Kingdom which was 1 in 3600 [6].

During the first year of their life, the patients with infantile type present with polyuria, polydipsia, vitamin D resistance rickets, and hypochloremic metabolic acidosis [7]. Moreover, during the second year, they develop cystine deposition in the cornea [8], and at the end of the first decade, $75 \%$ of them develop hypothyroidism [9] and pancreatic dysfunction [10]. Subsequently, in the second or third decades, the child has normal intelligence $[10,11]$ and they may develop cerebral atrophy during the older lifetime [12]. Additionally, few of them suffers with hypopigmentation, blue eyes, blond hair [13], craves for spicy and salty food [14]. Finally, a severe form of failure to thrive is the cardinal feature of infantile cystinosis [15].

The followings are diagnostic methods:

1. Cystine level in the Leukocyte which is the gold standard [16]

2. Corneal crystallization by corneal examination under slit lamp [17].

3. Genetic analysis searching for CTNS gene [18].

4. Antenatal investigation of chorionic villous tissue or amniotic fluid [19].

The management is mostly supportive. Moreover, cysteamine (oral or eye drop) is the specific treatment 
for lowering intralysosomal cystine [20]. Although the life-span of the patients will be prolonged by the usage of cysteamine, they will suffer from significant problems. Additionally, the study of Cherqui [21] showed with treatments mean survival age of 28 years. Hematopoietic stem cell transplantation may be the next step for the treatment of cystinosis in the future [22].

The purpose of our study was to evaluate the clinical presentations and outcome - mortality and morbidity of infantile nephropathic cystinosis among patients seen in the Department of Chronic Diseases in Sulaimani Pediatric Teaching Hospital.

\section{METHODS AND MATERIALS}

A retrospective cohort study was performed and all the patients (25 patients) who suffered from cystinosis were collected in the Sulaimani Pediatric Teaching Hospital, Kurdistan region, Iraq. The patients were from Sulaimani and other parts of Iraq whom recorded in the Department of Metabolic and Endocrine Diseases of Sulaimani Pediatric Teaching Hospital during May 1, 2014, to Jun 1, 2017. We collected the biographical data of the patients with their clinical features including: age, gender, the age of presentation, the age of diagnosis, residency, ethnicity, consanguinity, family history, presentations and complications. For the diagnosis, we depended on the clinical symptoms and signs, and corneal crystallization which was being detected under the slit lamp by ophthalmologists.

Inclusion criteria: all cases of infantile nephropathic cystinosis.

Exclusion criteria: all cases of juvenile and ocular cystinosis.

The (IBM SPSS Statistics 21) statistical program was used for the analysis of the data including descriptive and inferential statistical methods. Furthermore, we considered the P-value of $\leq 0.05$ to be statistically significant.

\section{RESULT}

The genders of the patients were 13 (52\%) females and 12 (48\%) males. The patients' age ranged from 1 to 12 years with a mean of 6.25 years $-1(4 \%)$ was 11 months, 8 (32\%) was between 13 months to 5 years, and 16 (64\%) was older than 5 years. The residency of the patients was 8 (32\%) inside the city of Sulaimani and 17 (68\%) cases from outside of the city. 17 (68\%) of the patients were Arabic ethnic group and the other 8 (32\%) cases were Kurdish ethnic group.

The study also showed a positive consanguinity for 20 (80\%) of the patients. Moreover, there was a 19 (76\%) positive family history for the patients.

The distribution of the age at the presentation of symptoms and signs was as follows: five (20\%) younger than seven months, 15 (60\%) between seven to 12 months, and five (20\%) between 13 months to 24 months. Adding to that, there was a statistically highly significant relationship between the age at the presentation and symptoms and signs (P-value of $<0.001)$. Furthermore, the ages of the diagnosis were one (4\%) at three months, eight (32\%) was between seven to 12 months, 13 (52\%) was between 13 months to five years and three (12\%) was above five.
The clinical features of all patients (100\%) presented with polyuria, polydipsia, and failure to thrive. The other clinical features were as follows: 23 (92\%) had photophobia, 10 (40\%) had constipation and five (20\%) presented with blond hair.

The complications that happened in the patients were 24 (96\%) rickets, 14 (56\%) renal insufficiency, five (20\%) hypothyroidism, four (16\%) genu valgum, three (12\%) growth hormone deficiency, and three (12\%) end-stage renal disease. Additionally, $8 \%$ of the patients died due to end-stage renal failure and there was a statistically highly significant relationship between end-stage renal disease and death (P-value $<0.001)$.

The results showed a statistically very highly significant relationship between hypothyroidism and ethnicity ( $\mathrm{P}$ value $<0.001$ ) and all the patients with hypothyroidism were Kurdish but no Arabic ethnic group. Moreover, there was also a statistically significant relationship (Pvalue $=0.042$ ) between renal insufficiency and ethnicity - Kurdish ethnic group suffered more (Table 1).

Table 1: shows a statistically very highly significant relationship between hypothyroidism and ethnicity, and a statistical significant relationship between renal insufficiency and ethnicity

\begin{tabular}{|c|c|c|c|c|}
\hline \multirow{2}{*}{\multicolumn{2}{|c|}{ Complications }} & \multicolumn{2}{|c|}{ Ethnicity } & \multirow{2}{*}{ P-values } \\
\hline & & Kurdish & Arabic & \\
\hline \multirow{3}{*}{$\begin{array}{l}\text { Hypothyroidis } \\
\text { m }\end{array}$} & Yes & 5 & 0 & \multirow{3}{*}{$<0.001$} \\
\hline & No & 3 & 17 & \\
\hline & Total & 8 & 17 & \\
\hline \multirow{3}{*}{$\begin{array}{c}\text { Renal } \\
\text { insufficiency }\end{array}$} & Yes & 7 & 7 & \multirow{3}{*}{0.042} \\
\hline & No & 1 & 10 & \\
\hline & Total & 8 & 17 & \\
\hline
\end{tabular}

\section{DISCUSSION}

The Female: Male ratio in the studies of Nakhaii et al. [23] in Iran and A.Azat [17] in Baghdad was showed an increased incidence of cystinosis among females (Female: Male ratio of 1:1.94). Moreover, the results of our study showed a slightly increased incidence among the females with a Male: Female ratio of (0.92). Furthermore, the ages of infantile nephropathic cystinosis in our study (ranged 1-12 years, mean= 6.25) were compatible with the studies performed in Turkey [24] (ranged 1.5 - 12 years) and the study of Shahkarami et al. [25] in Iran (ranged 7 months - 11 years). Sequentially, the age of the first presentation in our study was (mean=0.8 year, ranged $0.25-1$ year) which was about the same as found in the study of A.Azat [17] in Baghdad (mean = 1.1 years, ranged 0.3-3.5 years). Additionally, the study also showed a mean age at the time of diagnosis of 2.25 years (ranged $0.5-7$ years) which is twice less than the study of A.Azat [17] in Baghdad (mean $=4$ years, ranged $0.6-12$ years), while the study of Bertholet-Thomas et al [26] in developed countries showed about the same result (mean $=1.3$ years, ranged $0.9-7$ years).

The family history of the disease will increase the chances of occurrence of cystinosis as well as a positive 
parental consanguinity because the mode of inheritance is autosomal recessive. Our study showed a positive family history and consanguinity of $76 \%$ and $80 \%$ respectively. These results are compatible with the study performed in Baghdad [17] (37.9\% and 86.2\% positive family history and consanguinity respectively), and the study of Shahkarami et al. [25] in Iran (92\% positive consanguinity).

There are common clinical presentations among patients with infantile nephropathic cystinosis [27]. The present study and the study of A.Azat [17] in Baghdad and Shahkarami et al [25] in Iran all showed a 100\% presentation of polyuria, polydipsia, and failure to thrive. Furthermore, there are features that are not same in different populations. Our study showed a $40 \%$ frequency of constipation which differs from the study of A.Azat [17] in which all patients were presenting with constipation. The results in our Study may be explained by the early diagnosis and management of the patients in our population.

Other clinical presentations included photophobia which found in (92\%) patients in our study. The result is near to the study result of A.Azat [17] (photophobia = 62\%). Reasonably, photophobia is a common sign of infantile cystinosis with an onset at toddler ages due to the deposition of cystine crystal in the cornea. The study also showed a $20 \%$ frequency of blond hair occurrence which is about the twice less than to what is found by Chiaverini et al. [28] (40.7\%). The change in hair color may be due to an alteration of melanogenesis [29].

The cystinosis complicated by many complications including rickets, renal insufficiency, hypothyroidism, end-stage renal disease, genu valgum, and growth hormone deficiencies [17]. Our study showed a 96\% patients with rickets which is about the same of what is mentioned by Shahkarami et al. [25] (88\%) and A.Azat [17] (75\%). Rickets is one of the early complications of infantile cystinosis due to renal tubular Fanconi syndrome.

Renal insufficiency is another complication that occurred in $56 \%$ of our patients which is near to the results found by A.Azat [17] (41.3\%) and Doğan et al. [24] (36.3\%). Moreover, Hypothyroidism was $20 \%$ in our study while its frequency was $3.44 \%$ in the study of A.Azat [17]. This may be explained by the age difference between both studies - the mean age in our study was 6.25 years while that of the study of A.Azat [17] was at 4 years - because the occurrence of hypothyroidism is at the end of first decade of life [9].

The end-stage renal disease is a serious complication of cystinosis. Our study showed a $12 \%$ occurrence which is compatible with the study of A.Azat [17] (13.79\%) while differs from the study of Bertholet-Thomas et al. [26] in the developed countries (37.4\%). One explanation may be due to that, although there is a clinical improvement in the usage of cysteamine, most of the patients develop the end-stage renal disease at second or third decades of their life [9]. Furthermore, the study of Bertholet-Thomas et al. [26] used a large sample size (104 patients) with a mean age of 13 years (ranged 1.2 - 38.6 years) compared to the small sample size in our study.

Genu valgum was considered in the studies that concerned with bone problems. The study of Bacchetta et al. [30] who took only three patients of cystinosis who had bone problems, two of them had genu valgum. Furthermore, patients with genu valgum were $30.4 \%$ in the study of Besouw [31] who took 23 patients with cystinosis who had bone problems. Meanwhile, the present study showed $16 \%$ genu valgum in our patients. One of the explanations of bone diseases in cystinosis and Fanconi syndrome is the presence of the reduction in the abilities of osteoblast precursor cells to transform into mature osteoblast that capable of osteoid production and mineralization [32].

Growth hormone deficiency is a lesser complication in infantile cystinosis. The study of Dyck et al. [33] showed only one patient who complicated with deficiency of growth hormone. Conversely, our study showed a $12 \%$ frequency of growth hormone deficiency. The explanation for growth hormone deficiency may be due to the fact that there is a high urinary excretion of growth hormone and IGF-1 [34]. Additionally, the high frequency of growth hormone deficiency in our study may be due to alteration in the timing of growth hormone peak after stimulation test, thence, may indicate a subclinical alteration in its secretion. Moreover, diabetes mellitus occurred (25\%) in the study of Robert et al. [35] while we did not have any patients with diabetes mellitus (0\%). This may be caused by the fact that diabetes mellitus occurs during adolescence or adulthood [1] and the mean age in the study of Robert et al. [35] was 22.1 years.

There was a statistically significant relationship between the mortality ( $8 \%$ in our study) and end-stage renal failure (P-value was $<0.001)$, and all the patients $(100 \%)$ died in our study had the end-stage renal failure. Moreover, the study of A.Azat [17] showed 20.8\% mortality with a $50 \%$ chronic renal failure as a cause. In contrast, the study of Bertholet-Thomas et al. [26] showed $0 \%$ mortality rate in developed countries because they performed a renal transplant for most of their patients (31/38). This study and A.Azat [17] did not perform a renal transplant for the patients with end-stage renal failure which is usually indicated [36].

Finally, our study showed a statistically significant relationship between renal insufficiency and Kurdish ethnic group $(\mathrm{P}$-value $=0.042)$, and a statistically very highly significant relationship between hypothyroidism and Kurdish ethnic group (P-value <0.001) (Table 1). This may be explained by the variation in the ages of both Kurdish and Arabic ethnic groups - Mean ages were 7.75 years and 4.75 years for Kurdish and Arabic ethnic groups respectively.

\section{CONCLUSION}

The present study shows a high incidence between parents with a positive consanguinity and those patients 
who had a positive family history of cystinosis. Furthermore, the explanation for the high incidence among patients with positive family history can be due to poor medical counseling, antenatal and postnatal screening tests.

The delay in its diagnosis was due to atypical clinical presentation and unavailability of specific genetic tests. Moreover, the early onset complications were due to the inadequacy of specific medications.

\section{ACKNOWLEDGEMENT}

We are very grateful to the help of the staffs of the Department of Metabolic and Endocrine Diseases of Sulaimani Pediatric Teaching Hospital.

\section{REFERENCES}

[1] W. A. Gahl, J. G. Thoene, J. A. Schneider. "Cystinosis". N Engl J Med, Vol.347, PP.111-121, 2002.

[2] G. Nesterova, W. A. Gahl. "Cystinosis: the evolution of a treatable disease". Pediatr Nephrol. Vol. 28, PP. 51-59, 2013. doi:10.1007/s00467-012-2242-5.

[3] E. Levtchenko, L. van den Heuvel, F. Emma et al. "Clinical utility gene card for: cystinosis". Eur J Hum Genet., Vol. 22, PP. e1-e3, 2014. doi:10.1038/ejhg.2013.204

[4] M. Town, G. Jean, S. Cherqui et al. "A novel gene encoding an integral membrane protein is mutated in nephropathic cystinosis". Nat Genet, Vol. 18, PP. 319-324, 1998.

[5] G. Nesterova, W. Gahl. "Nephropathic cystinosis: late complications of a multisystemic disease". Pediatr Nephrol, Vol. 23(6), PP. 863-878, Jun. 2008. doi: 10.1007/s00467-007-0650-8

[6] A. C. Hutchesson, S. Bundey, M. A. Preece et al. "A comparison of disease and gene frequencies of inborn errors of metabolism among different ethnic groups in the West Midlands, UK”. J Med Genet, Vol. 35, PP. 366-370, 1998.

[7] M. Greco, M. Brugnara, M. Zaffanello et al. "Long-term outcome of nephropathic cystinosis: a 20-year single-center experience". Pediatr Nephrol. Vol. 25, PP. 2459-2467, 2010. doi: 10.1007/s00467-010-1641-8

[8] E. Tsilou, M. Zhou, W. Gahl et al. "Ophthalmic manifestations and histopathology of infantile nephropathic cystinosis: report of a case and review of the literature". Surv Ophthalmol, Vol. 52, PP. 97-105, 2007. doi: 10.1016/j.survophthal.2006.10.006

[9] W. A. Gahl, E. M. Kuehl, F. Iwata et al. "Corneal crystals in nephropathic cystinosis: natural history and treatment with cysteamine eyedrops". Mol Genet Metab. Vol. 71, PP. 100-120. 2000. doi: https://doi.org/10.1006/mgme.2000.3062

[10] J. J. Robert, M. J. Tête, G. Guest et al. "Diabetes mellitus in patients with infantile cystinosis after renal transplantation". Pediatr Nephrol, Vol. 13, PP. 524-529, 1999. doi: https://doi.org/10.1007/s004670050651

[11] S. Bava, R. J. Theilmann, M Sach et al. "Developmental changes in cerebral white matter microstructure in a disorder of lysosomal storage". Cortex, Vol. 46, PP. 206-216, 2010. doi: https://doi.org/10.1016/j.cortex.2009.03.008

[12] M. Broyer, M. J. Tete, G. Guest et al. "Clinical polymorphism of cystinosis encephalopathy. Results of treatment with cysteamine". J Inherit Metab Dis, Vol. 19, PP. 65-75, 1996. doi: https://doi.org/10.1007/BF01799350

[13] G. Guillet, B. Sassolas, S. Fromentoux et al. "Skin storage of cystine and premature skin ageing in cystinosis". Lancet, Vol. 352, PP. 1444-1445, 1998. doi: http://dx.doi.org/10.1016/S01406736(05)61267-6

[14] M. Besouw, E. Levtchenko. "Growth retardation in children with cystinosis". Minerva Pediatr, Vol. 62, PP. 307e14, Jun. 2010.

[15] K. J. V. Stralen, F. Emma, K. J. Jager et al. "Improvement in the renal prognosis in nephropathic cystinosis". Clin J Am Soc Nephrol. Vol. 6, PP. 2485-2491. 2011. doi: 10.2215/CJN.02000311

[16] R. G. Oshima, R. C. Willis, C. E. Furlong et al. "Binding assays for amino acids. The utilization of a cystine binding protein from Escherichia coli for the determination of acid-soluble cystine in small physiological samples". J Biol Chem, Vol. 249, PP. 60336039, 1974
[17] N. F. A.Azat. "Natural History of Symptomatically Treated Children with Cystinosis". The Iraqi Postgraduate Medical Journal, Vol.11, PP. 253-257, 2012.

[18] M. J. Wilmer, J. P. Schoeber, L. P. van den Heuvel et al "Cystinosis: practical tools for diagnosis and treatment". Pediatr Nephrol. Vol. 26, PP. 205-215, Feb. 2011. doi: 10.1007/s00467010-1627-6

[19] M. Jackson, E. Young. "Prenatal diagnosis of cystinosis by quantitative measurement of cystine in chorionic villi and cultured cells". Prenat Diagn, Vol. 25, PP. 1045-1047, Nov. 2005. doi: 10.1002/pd.1249

[20] G. Nesterova, W. Gahl. "Cystinosis: the evolution of a treatable disease". Pediatr Nephrol, Vol. 28, PP. 51-59, Jan. 2013 doi: 10.1007/s00467-012-2242-5

[21] S. Cherqui. "Cysteamine therapy: a treatment for cystinosis, not a cure". Kidney Int, Vol. 81, PP. 127-129, 2012. doi: 10.1038/ki.2011.301

[22] J. G. Thoene. "A review of the role of enhanced apoptosis in the pathophysiology of cystinosis". Mol Genet Metab, Vol. 92, PP. 292-298, 2007.

[23] S. Nakhaii, N. Hooman, H. Otoukesh. "Gastrointestinal Manifestations of Nephropathic Cystinosis in Children". Iran J Kidney Dis, Vol. 3, PP. 218-221, Oct. 2009

[24] M. Doğan, K. Bulan, S. Kaba et al. "Cystinosis in Eastern Turkey". J Pediatr Endocrinol Metab, Vol. 1;29, PP. 965-969, Aug. 2016. doi: 10.1515/jpem-2014-0477

[25] S. Shahkarami , H. Galehdari, A. Ahmadzadeh et al. "The first Molecular genetics analysis of individuals suffering from nephropatic cystinosis in the Southwestern Iran". Nefrología, Vol. 33, PP. 308-315, $2013 . \quad$ doi: 10.3265/Nefrologia.pre2012.Sep.11558

[26] A. Bertholet-Thomas, J. Berthiller, V. Tasic et al. "Worldwide view of nephropathic cystinosis: results from a survey from 30 countries". BMC Nephrol, Vol. 18, PP. 210, Jul. 2017. doi: 10.1186/s12882-017-0633-3

[27] M. Greco, M. Brugnara, M. Zaffanello et al. "Long-term outcome of nephropathic cystinosis: a 20-year single-center experience". Pediatr Nephrol, Vol. 25, PP. 2459-2467, 2010.doi: 10.1007/s00467-010-1641-8

[28] C. Chiaverini, L. Sillard, E. Flori, S. Ito, S. Briganti, K. Wakamatsu et al. "Cystinosis is a melanosomal protein that regulates melanin synthesis". FASEB journal, Vol. 26, PP. 37793789, 2012. doi: fj.11-201376v1 26/9/3779

[29] G. Guillet, B. Sassolas, S. Fromentoux et al. "Skin storage of cystine and premature skin ageing in cystinosis". Lancet, Vol. 352, PP. 1444-1445, Oct. 1988. doi: https://doi.org/10.1016/S0140-6736(05)61267-6

[30] J. Bacchetta, M. Greco, A. Bertholet-Thomas et al. "Skeletal implications and management of cystinosis: three case $r$ eports and Literature review". Bonekey Rep, Vol. 5, PP. 828, Aug. 2016. doi: 10.1038/bonekey.2016.55.

[31] M Besouw. "Bone problems in patients with cystinosis". 47th annual meeting of the eauropian society for pediatric nephrology (ESPN), Porto, Portugal, 2014.

[32] A. Conforti, A. Taranta, S. Biagini et al. "Cysteamine treatment restores the in vitro ability to differentiate along the osteoblastic lineage of mesenchymal stromal cells isolated from bone marrow of a cystinotic patient". J Transl Med. Vol. 13, PP. 143, May 2015. doi: 10.1186/s12967-015-0494-0

[33] M. T. P. Besouw, M. V. Dyck, I. Francois et al. "Detailed studies of growth hormone secretion in cystinosis patients". Pediatr Nephrol, Vol. 27, PP. 2123-2127. doi: 10.1007/s00467-0122213-x

[34] D. S. Theodoropoulos, T. H. Shawker, C. Heinrichs et al. "Medullary nephrocalcinosis in nephropathic cystinosis". Pediatr Nephrol, Vol. 9, PP. 412-418, Aug. 1995

[35] J.-J. Robert, M.-J. Tete, G. Guest et al. "Diabetus mellitus in patients with infantile cystinosis after renal transplantation". Pediatric nephrology, Vol. 13, PP. 524-529, Jul. 1999. doi: https://doi.org/10.1007/s004670050651

[36] F. Emma, G. Nesterova, C. Langman et al. "Nephropathic cystinosis: an international consensus document". Nephrol Dial $\begin{array}{lllll}\text { Transplant. } & \text { Vol. 29, PP. } & \text { 87-94. } 2014 .\end{array}$ http://ndt.oxfordjournals.org/content/29/suppl_4/iv87. 